\title{
Laser transmitter for CubeSat-class applications
}

\author{
Jonathan R. Crabb*a,b, G. Stevens ${ }^{\mathrm{b}}$, C. Michie ${ }^{\mathrm{b}}$, W. Johnstone ${ }^{\mathrm{b}}$ and E. Kehayas ${ }^{\mathrm{a}}$ \\ ${ }^{a}$ Gooch \& Housego (Torquay), Broomhill Way, Torquay, Devon, TQ2 7QL; \\ ${ }^{b}$ Department of Electronic Engineering, University of Strathclyde, Royal College Building, \\ 204 George Street, Glasgow, G1 1XW, UK
}

\begin{abstract}
Laser communications onboard CubeSats is an emerging technology for enabling high-speed space-based communication links. In this paper we present the development of a $25 \mathrm{~cm}^{3}$ and second iteration $0.3 \mathrm{U}$ CubeSat-class laser transmitter operating at data rates of up to $500 \mathrm{Mbps}$ using OOK modulation and an output power of up to $300 \mathrm{~mW}$ over the entire Cband. We present results of the development and characterization of the transmitter. From this testing the design will be demonstrated up to TRL 4/5 with the view for future qualification work and electronics integration.
\end{abstract}

Keywords: Fiber optics, laser transmitters, communications, free space optics, satellite communications, space photonics, CubeSats

\section{INTRODUCTION}

Small satellites have long been identified as disruptive technologies for both scientific and commercial satellite missions. However, in recent times one of the major limiting factors affecting the scope of these missions is that of data transport due to strains on the RF spectrum ${ }^{1}$. Laser-based communications can overcome this problem by offering more compact, lighter terminals such that higher data rates can be provided to small satellite systems with a lower impact to the satellite bus. As part of the European Space Agency's ARTES program "Miniaturized Laser-Comm Transmitters for Small Satellite Platforms" Gooch \& Housego $(\mathrm{G} \& \mathrm{H})$ is developing laser communication transmitters (PERSEUS series) in the optical Cband for low power, low data rate $(300 \mathrm{~mW}, 1 \mathrm{Gbps})$ and high power, high data rate $(3 \mathrm{~W}, 10 \mathrm{Gbps})$ applications suitable for small-satellite and micro-satellite platforms. This leverages previous work by G\&H in which radiation resistant optical amplifiers for satellites have been developed up to a TRL of $7^{2-5}$.

In this paper we present the development of a new CubeSat-class laser transmitter based on a directly-modulated DFB laser and fiber amplifier. Two laser transmitter variants were designed, one bespoke design for system integration into the CubeCAT (REF) and a second module suitable for integration on a standard CubeSat footprint, occupying a volume of $0.3 \mathrm{U}(96 \times 96 \times 30 \mathrm{~mm})$. The current laser transmitter only incorporates the laser engine with no control electronics, is designed to operate at an output power up to $400 \mathrm{~mW}$ over the entire C-band and provide data rates up to $1 \mathrm{Gbps}$ using OOK modulation. As is with most CubeSat modules, COTS components which have a proven heritage in high-reliability applications are used to keep costs and developmental costs down. In the module presented, COTS optical components which have a heritage within G\&H's high-reliability products are used to ensure that a high level of quality is still achieved at an affordable cost. In addition, results verifying the optical and electronic design will be presented. Optical and electronic results will be presented characterizing the transmitter optical and communications performance in turn demonstrating this technology to TRL $4 / 5$.

\subsection{Laser Transmitter Specification}

Presented in Table 1 is the product specification of the laser transmitter reported, PERSEUS-LP-LE-C-LR (Low PowerLaser Engine-C band-low data rate), in this paper. It is designed to provide a minimum output power of $150 \mathrm{~mW}$ over the optical C-band (CW) with a minimum data rate of $1 \mathrm{Gbps}$, scalable up to $2.5 \mathrm{Gbps}$.

*j.crabb@goochandhousego.com, +44 (0)1803 611744, goochandhousego.com 
Table 1: PERSEUS-LP-LE-C-LR laser transmitter specification

\begin{tabular}{|l|c|c|c|}
\hline Parameter & Value & Unit & Notes \\
\hline Wavelength & $1535-1560$ & $\mathrm{~nm}$ & C-band \\
\hline Source Intrinsic Linewidth & $<500$ & $\mathrm{MHz}$ & $\mathrm{CW}$ \\
\hline Min Required EOL Output Power & 0.2 & $\mathrm{~W}$ & CubeCAT requirement \\
\hline Max. Output Power & 0.4 & $\mathrm{~W}$ & - \\
\hline Data Rate & Min: 1 & $\mathrm{Gbps}$ & Scalable to 2.5 Gbps \\
\hline Modulation Depth & $>20$ & $\mathrm{~dB}$ & @ 1 Gbps \\
\hline Data Encoding Scheme & OOK & - & - \\
\hline Fiber type & $\mathrm{SM}$ & - & non-PM \\
\hline OSNR & $>20$ & $\mathrm{~dB}$ & - \\
\hline Output interface & Bare fiber & - & - \\
\hline Output fiber MFD & $10.4 \pm 0.5$ & $\mu \mathrm{m}$ & - \\
\hline Rise Time & 100 & $\mathrm{ps}$ & $10-90 \%$. \\
\hline Fall Time & -23 & $\mathrm{~dB}$ & - \\
\hline TX Reflection Isolation & 30 & $\mathrm{~dB}$ & - \\
\hline Side Mode Suppression Ratio & 25 & $\mathrm{~cm}^{3}$ & CubeCAT \\
\hline \multirow{2}{*}{ Volume } & $96 \times 96 \times 30$ & $\mathrm{~mm}$ & $0.3 \mathrm{U}$ \\
\cline { 2 - 4 } & $<150$ & $\mathrm{~g}$ & CubeCAT \\
\hline Mass & Min: 10 & $\%$ & Target: $15 \%$ \\
\hline
\end{tabular}

\section{LASER TRANSMITTER DESIGN}

\subsection{Optical Architecture}

The laser transmitter presented is designed to operate in a simple master oscillator power amplifier (MOPA) architecture as shown in Figure 1. The master oscillator (shown as LTX) is a directly modulated G\&H distributed feedback (DFB) laser driven using a $50 \Omega$ bias-T. This allows for an AC modulation current to be overlaid on a DC bias current (near $\mathrm{I}_{\mathrm{th}}$ ); this in turn allows for the highest extinction ratio available. This is then fed into a high-efficiency EDFA which provides the required optical amplification and lowest noise solution. The amplifier architecture was based on core-pumped design in order to maintain low power consumption. Supplied interfaces are shown in dashed boxes

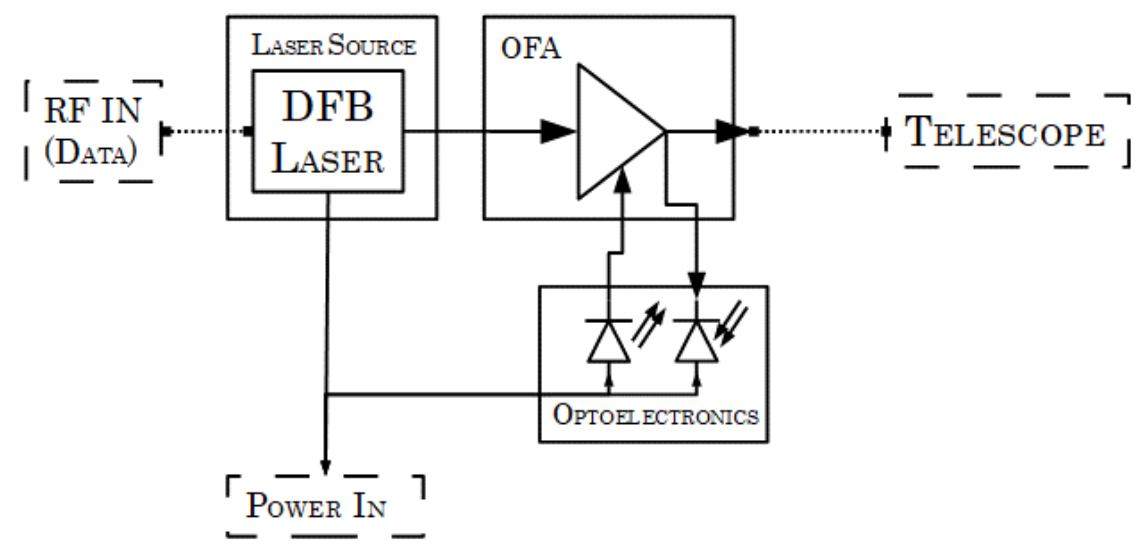

Figure 1: PERSEUS-LP-LE-C-LR Optical Architecture 
By using previous documented work at $\mathrm{G} \& \mathrm{H}^{2-5}$, optical component selection was based upon radiation hardness performance, reliability, commercial availability and overall impact on the system parameters in terms of SWaP (size, weight and power). For this reason COTS components which have been used in previous work ${ }^{2-5}$ were selected to provide component qualification without the need for costly and lengthy external qualification campaigns. G\&H's previous work allowed for the selection of a COTS DFB laser, fused fiber couplers and WDMs from G\&H. For parts not previously used components were selected such that previous environmental testing to terrestrial standards had already be completed. Qualification to terrestrial standards as a minimum requirement suggest these components are good candidates for future qualification in order to reach TRL $>6$.

\subsection{Mechanical Design}

As CubeSats are designed to be extremely small, a major challenge was to design the laser transmitter such that it would fit into as small a volume as possible (thus providing minimal impact to the system bus), whilst still fitting to the CubeSat standard footprint $(96 \times 96 \mathrm{~mm})$. However, for the CubeCAT project this volume was modified and reduced further such that a telescope and control electronics could be integrated into a total volume of 2 U. Figure 2 shows the mechanical housing for the CubeCAT laser engine, showing the fiber exit and electrical connection through a 16-way high-reliability connector. Not shown is the RF interface which is through a flying $50 \Omega$ U.FL lead which exits the slot circled in red. The total volume is $25 \mathrm{~cm}^{3}$. To ensure high-reliability performance of fiber optical components, component layout is key to ensure that the minimum bend radius encountered by the optical fiber was kept within G\&H design rules, and to minimize volume.

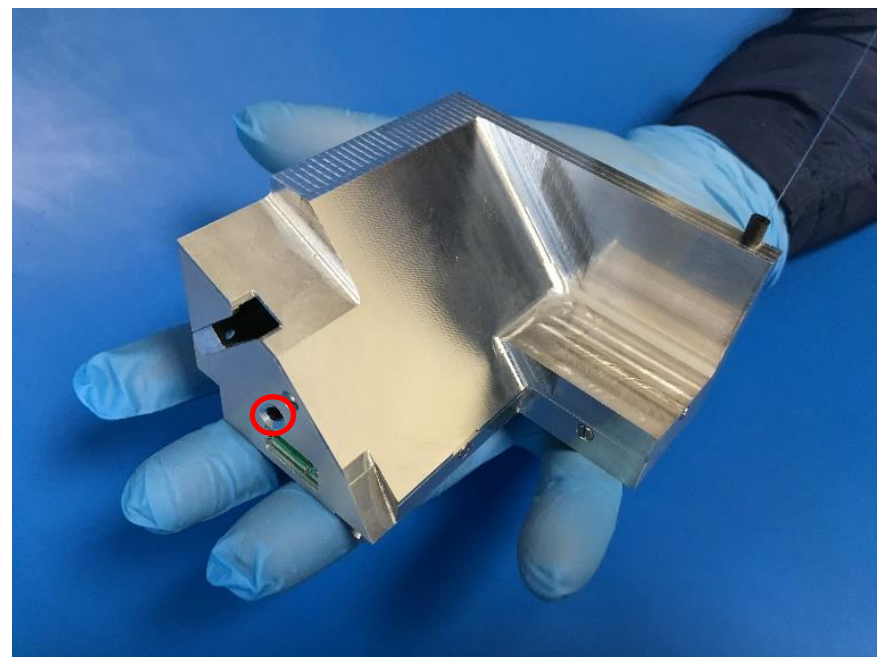

Figure 2: CubeCAT mechanical structure. Not shows is the RF (data) connection is supplied by a $50 \Omega$ U.FL fly lead. The RF port for the fly lead is circled in red.

Shown in Figure 3 is an iterated mechanical design which shows the PERSEUS-LP-LE-C-LR module now laid out to use the standard CubeSat footprint $(96$ x $96 \mathrm{~mm})$. The design in Figure 3 occupies a volume of 96 x 96 x $28 \mathrm{~mm}$ (approx.0.3 U) and is designed to allow for ease of high volume assembly by allowing for separate assembly of the opto-electronics and fiber optics. 


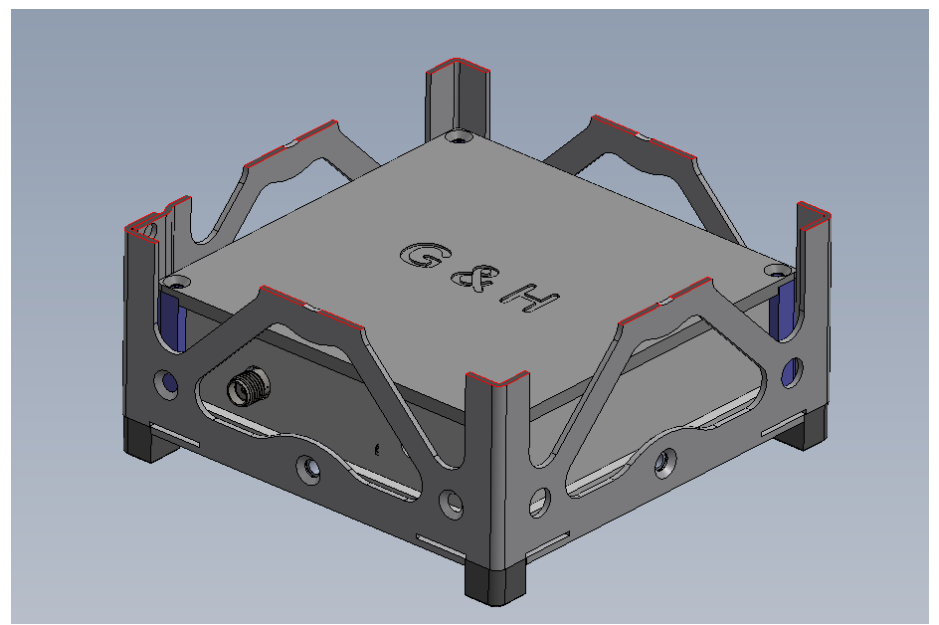

Figure 3: PERSEUS-LP-LE-C-LR mechanical CAD design set out in a 1U CubeSat skeleton

\section{OPTICAL PERFORMANCE}

\subsection{Optical Simulation}

To assess the optical performance of the system, the laser transmitter was first theoretically modelled. The first stage of simulation was to assess the CW performance of the laser transmitter which in turn was verified using empirical data. The aim of this simulation was to predict the L-I performance of the module and show that the output power requirements are met at End of Life (EOL). The simulation assumed an input power of $+10 \mathrm{dBm}$ at a wavelength of $1550 \mathrm{~nm}$. Shown in Figure 4 are the simulated and empirical results of the CW performance of the PERSEUS-LP-LE-C-LR module. It shows little variation between simulated and measured values with a maximum difference observed of $0.22 \mathrm{~dB}$ at high pump drive currents which is within the margin of error. Thus it can be concluded that the simulation holds for a real system.

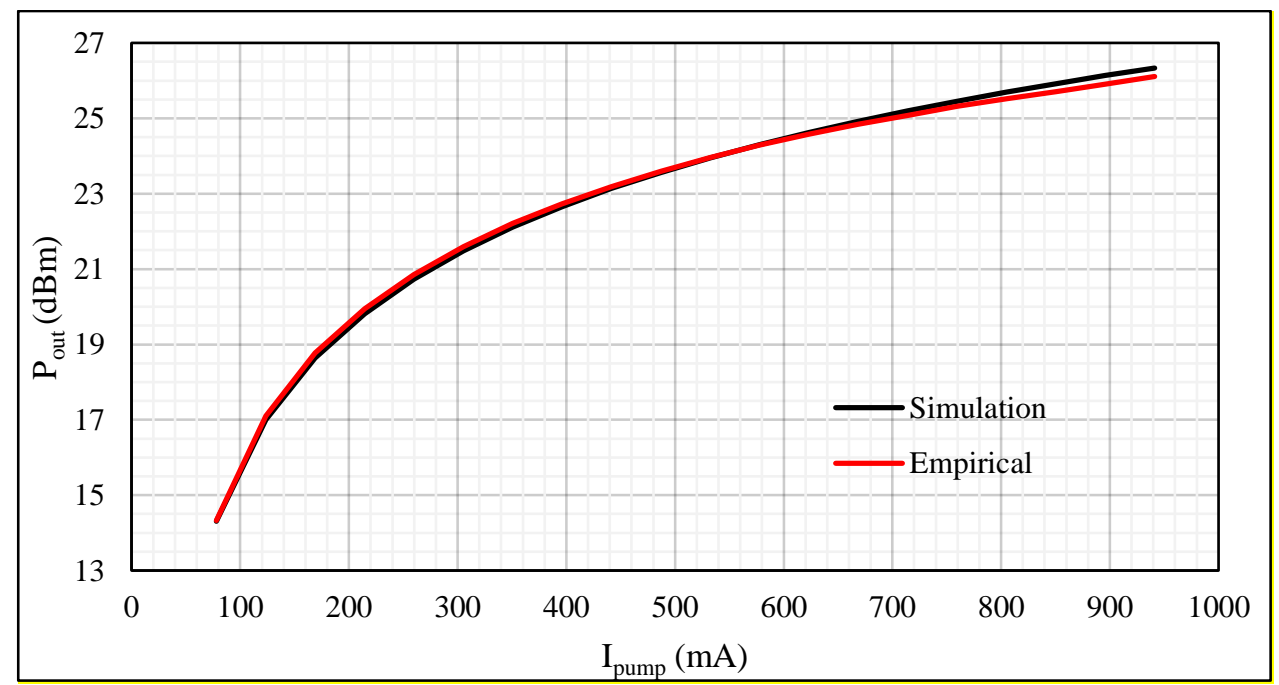

Figure 4: Simulated (black) and Measured (red) L-I curve for the CW performance of the PERSEUS-LP-LE-C-LR module.

Figure 4 also verifies the output power performance up to output powers of $400 \mathrm{~mW}(+26 \mathrm{dBm})$ at a maximum pump drive current of $940 \mathrm{~mA}$. In addition, it shows that the module can easily meet the minimum power requirement of 200 $\mathrm{mW}(23 \mathrm{dBm})$ at a good efficiency (approx. $15 \%$ wall-plug efficiency) whilst leaving a margin of $3.3 \mathrm{~dB}$ for degradation over life. 
The second stage of simulation was to investigate the modulation current required for the DFB laser at a data rate of $1 \mathrm{Gbps}$ passing through an optical amplifier. To assess the effect of modulation current, eye diagram analysis was used to assess the effect of modulation current on the eye opening. Three example eye diagrams are shown in Figure 5 at modulation currents of $4 \mathrm{~mA}$ (red), 10mA (dark yellow) and $20 \mathrm{~mA}$ (blue/grey). The simulation was set such that the DFB DC bias was set at $45 \mathrm{~mA}$ and threshold current at $50 \mathrm{~mA}$. This was to provide a large enough extinction ration between the ' 1 ' and ' 0 ' bit would be present such that a sensible bit discrimination level could be set. This can be seen in Figure 5 between the red (still below $\mathrm{I}_{\mathrm{th}}$ ) and dark yellow (above $\mathrm{I}_{\mathrm{th}}$ ) curves.

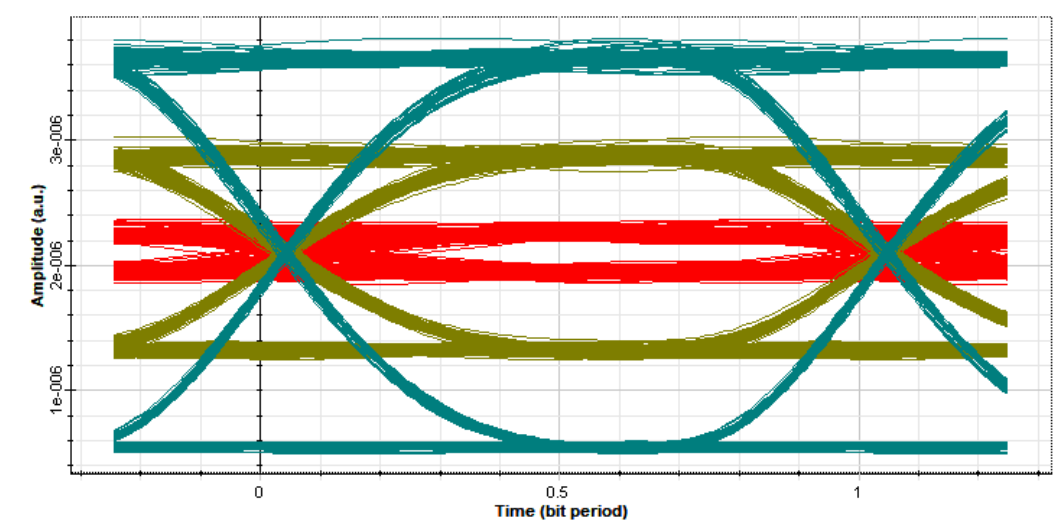

Figure 5: Simulated Eye diagrams for a DFB modulation current of $2 \mathrm{~mA}$ (red), $10 \mathrm{~mA}$ (dark yellow) and $20 \mathrm{~mA}$ (grey/blue) with a DFB Ibias $=45 \mathrm{~mA}$ and $\mathrm{I}_{\mathrm{th}}=50 \mathrm{~mA}$.

To quantitatively assess the impact of modulation current, a graph of eye opening factor, a ratio of the eye height (vertical opening of eye) to eye amplitude (difference between mean values of ' 1 ' and ' 0 ' values) versus modulation current can be plotted. This is shown in Figure 6. From Figure 6 it can be seen that as the modulation current is increased, the eye becomes more open as the modulation current is increased as expected. Note: No measurement was able at $2 \mathrm{~mA}$ due to the eye being too closed due to too much time jitter.

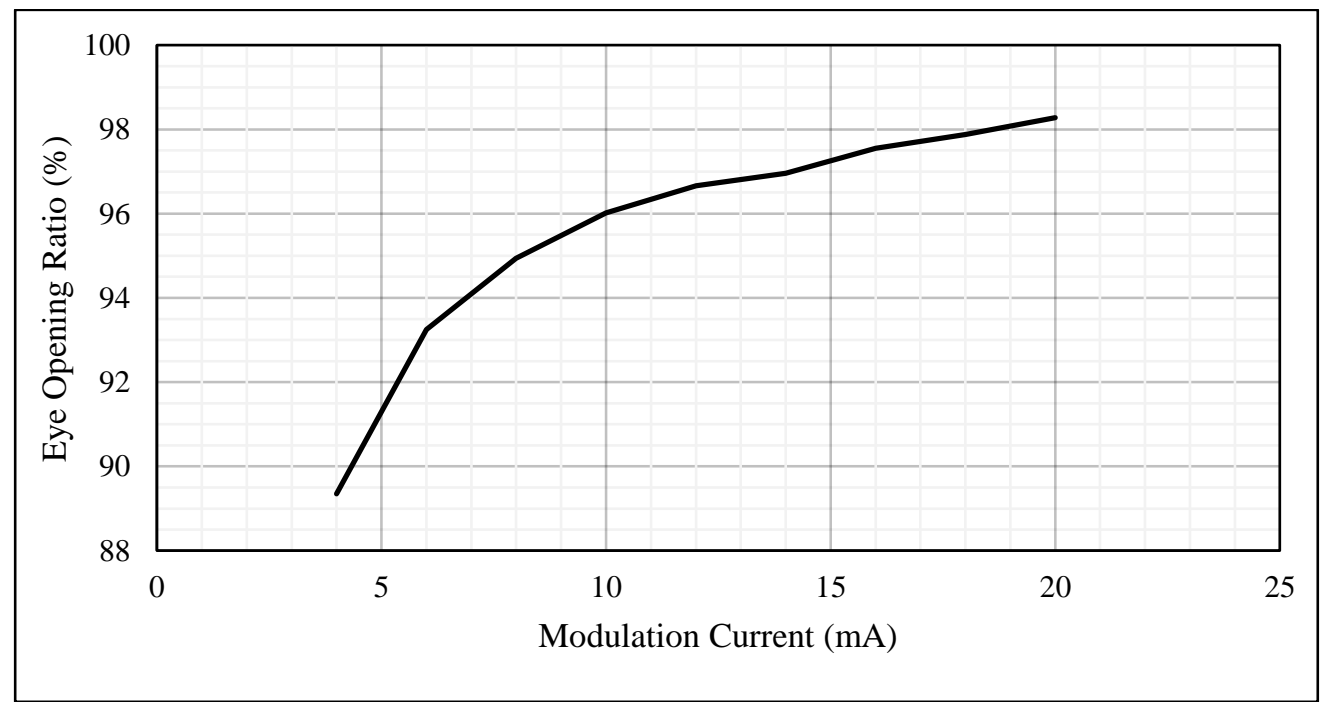

Figure 6: A graph of simulated eye opening factor versus modulation current.

In an ideal situation, as high a value of modulation current should be selected but in practice this is limited by the drive electronics. Based off the CubeCAT system requirements a modulation current of $10 \mathrm{~mA}$ is allowed. This in turn leads to an eye opening ratio of $96 \%$ which is acceptable on a system level. With a modulation current of $10 \mathrm{~mA}$ selected 
the laser transmitter was simulated to predict the average transmitted output power. It was assumed that a wavelength of $1550 \mathrm{~nm}$ was used and the amplifier was set to provide a gain of $20 \mathrm{~dB}$. From this simulation it was found that an average output power of $+23.3 \mathrm{dBm}$ is achievable. This relates to an average input power of $+3.3 \mathrm{dBm}$. Although not simulated, in the first set of simulations (Figure 4), the effect of input power is verified later at the breadboard stage.

\subsection{Optical test results}

Optical testing was completed to provide information on the laser transmitter output power performance in terms of pump power, input power and wavelength dependence. The results for the output power have already been presented and discussed in Figure 4.

As the simulations in Figure 4 are for an average input power of $+10 \mathrm{dBm}$, investigations into the amplifier output power against input power were performed. Measurements were made at input powers of $+3,+5$ and $+10 \mathrm{dBm}$, at a wavelength of $1550 \mathrm{~nm}$ and pump drive current of $450 \mathrm{~mA}$ (approximately half of the maximum drive current). The results are shown in Table 2. From Table 2 it can be seen that a drop of $\sim 1 \mathrm{~dB}$ of output power is observed over an input power decrease of $+7 \mathrm{dBm}$. This shows that the amplifier is saturated due to input power to some degree but the output power drops below the required output power of $+23 \mathrm{dBm}$. However, it is anticipated that an output power of $+25 \mathrm{dBm}$ at maximum pump current is achievable at an input power of $+3 \mathrm{dBm}$ (as predicted through simulation) due to more pump power being available.

Table 2: Average output power as a function of average input power of the laser transmitter.

\begin{tabular}{|c|c|}
\hline $\mathrm{P}_{\text {in }}(\mathrm{dBm})$ & $\mathrm{P}_{\text {out }}(\mathrm{dBm})$ \\
\hline 3 & 22.04 \\
\hline 5 & 22.69 \\
\hline 10 & 23.09 \\
\hline
\end{tabular}

The wavelength dependence of the output power and noise figure (NF) are reported in Figure 7. The laser transmitter was set such an output power of $+23 \mathrm{dBm}$ was achieved at $1550 \mathrm{~nm}$ and the wavelength then swept between 1530 and $1565 \mathrm{~nm}$.

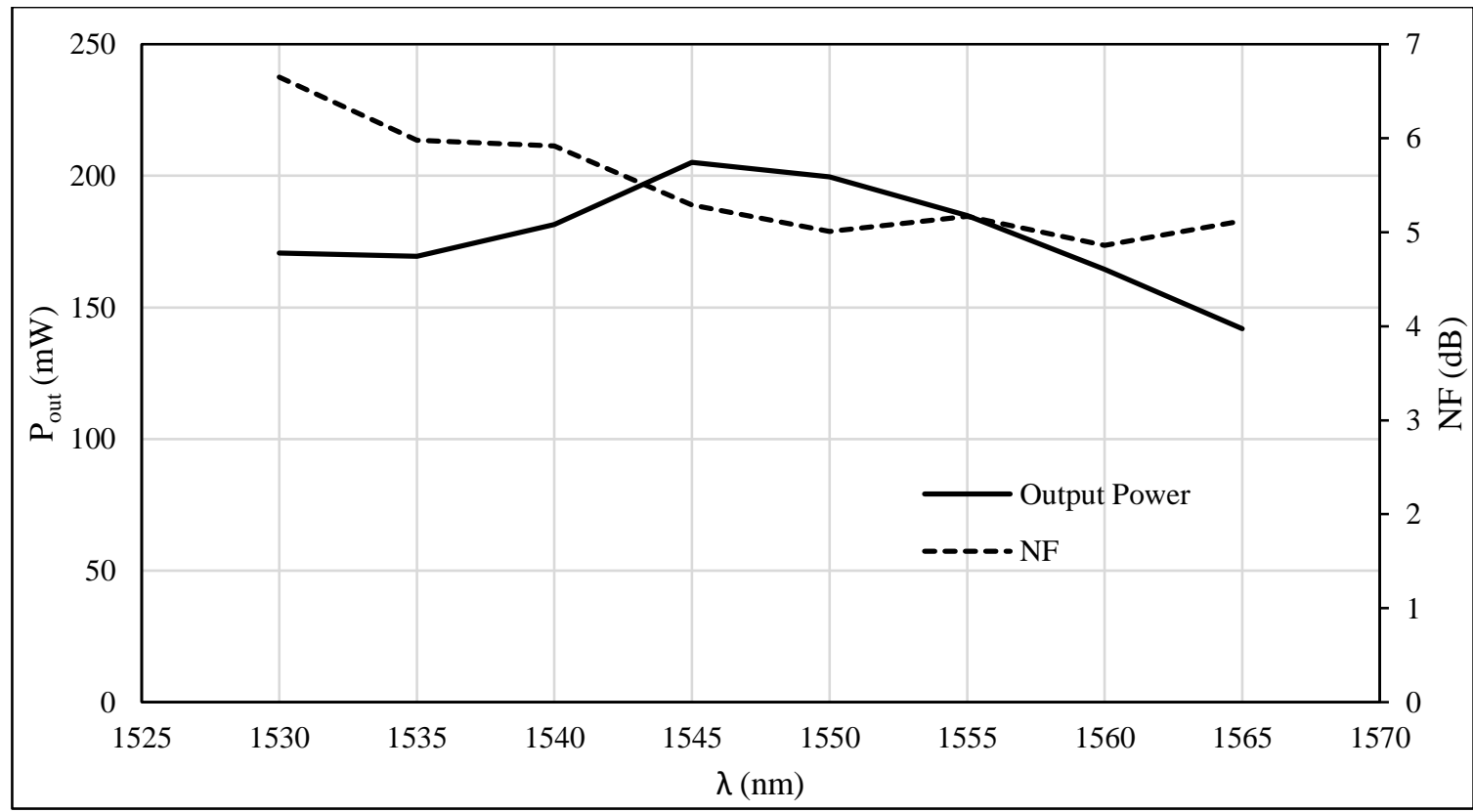

Figure 7: Average output power and noise figure as a function of wavelength. 
From Figure 7 it can be seen that a difference in output power of $63 \mathrm{~mW}$ exists and $1.8 \mathrm{~dB}$ in NF over the C-band. It is anticipated that the output power drop seen over the $\mathrm{C}$-band can be recovered by increasing the pump power as these measurements were taken at half the available pump power. At $1550 \mathrm{~nm}$ an output power of $200 \mathrm{~mW}$ is achieved with a noise figure of $5 \mathrm{~dB}$.

A laser transmitter breadboard was development and a preliminary assessment of the dynamic behavior of the system was performed by directly modulating the laser with $100 \mathrm{Mbps}$ and $500 \mathrm{Mbps}$ of data. The recorded eye diagrams are shown in Figure 8. Although a relatively good eye opening is seen in the eye diagram indicating a high modulation depth, significant ringing is present suggesting that there is a problem with the RF driving circuit or signal distortion caused by a mismatched interface. The time jitter present is thought to be from the measurement system and improper synchronization between the clock and received signal. From this data no BER measurements were attempted, as the measurement would show significant power penalty due to the pulse shape distortion. Further work is needed to provide better electrical interfacing to reduce the level of ringing observed and to maintain signal integrity. This will then allow a detailed analysis on the eye diagram and BER performance of the transmitter in a back-to-back configuration.

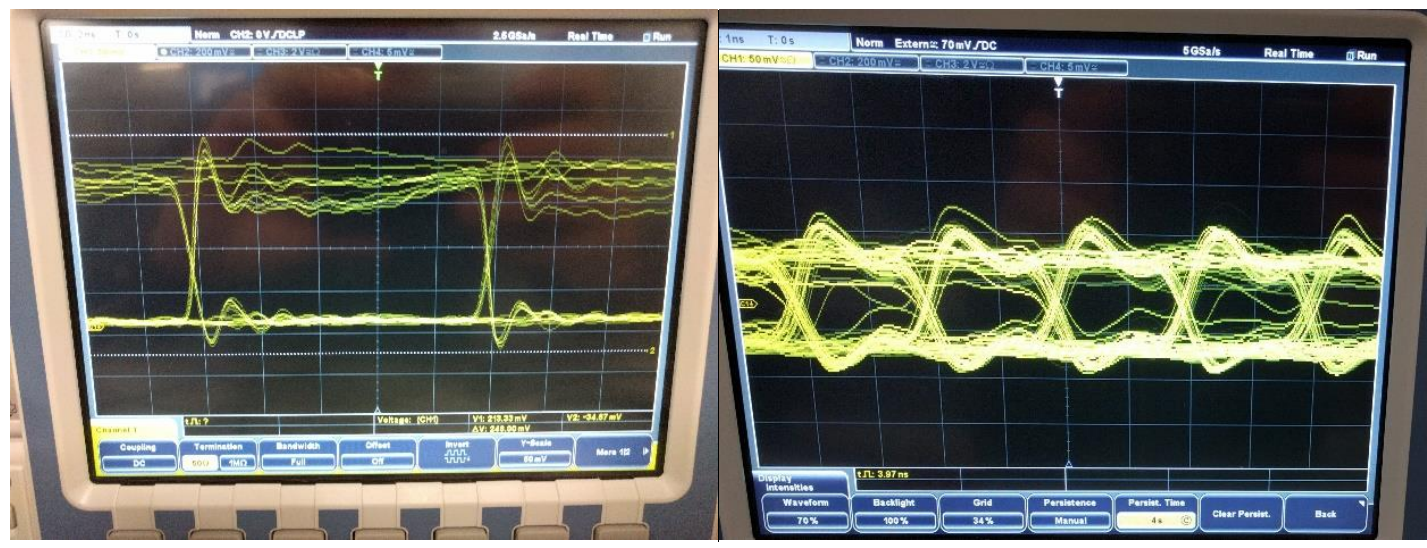

Figure 8: Photo of an Eye Diagram generated on an oscilloscope at $100 \mathrm{Mbps}$ (left) and $500 \mathrm{Mbps}$ (right).

\section{CONCLUSIONS}

To conclude we have reported a laser transmitter design for CubeSats that builds on G\&H work in high-reliability amplifiers and space-qualified photonic components. The laser transmitter has been simulated and experimentally demonstrated at a minimum output power of $200 \mathrm{~mW}$ across the C-band at a data rate of up to $500 \mathrm{Mbps}$. It shows good noise performance at $1550 \mathrm{~nm}$ with a NF of $5 \mathrm{~dB}$. The major design challenge was to design the module such that it could fit in a constrained volume for both the CubeCAT and in a standardized CubeSat footprint of 0.3 U. 1 Gbps operation has not yet been demonstrated but it is anticipated that this condition can be met with further work on the drive electronics and the test detection system, as the RF dynamic bandwidth of the laser source is more than capable to support such line rates.

In parallel to the activity presented in this paper we have also started development of a CubeSat-compliant driver and digital interface board to control the unit.

\section{ACKNOWLEDGMENTS}

The author wishes to acknowledge the funding support from ESA through the ARTES project "Miniaturised laser-comm transmitters for small satellite platforms" and ESPRC providing funding to the author through the "CDT in Applied Photonics". In addition the author wishes to acknowledge the CubeCAT project team at TNO and Hyperion Technologies B.V. who have provided initial specifications for this product under development. The author gratefully acknowledges the support received from his supervisors in supplying guidance in both the design and theory of the work in this paper. 


\section{REFERENCES}

[1] J. Farserotu and R. Prasad, "A survey of future broadband multimedia satellite systems, issues and trends," IEEE Commun. Mag., 38 (6), 128-133 (2000).

[2] L. Stampoulidis, E. Kehayas, M. Kehayas, G. Stevens, L. Henwood-Moroney, P. Hosking, and A. Robertson, "Radiation-hard Mid-power Booster Optical Fiber Amplifiers for High-speed Digital and Analogue Satellite Laser Communication Links," International Conference on Space Optics (ICSO) 2014.

[3] L. Stampoulidis, J. Edmunds, M. Kechagias, G. Stevens, J. Farzana, M. Welch, and E. Kehayas, "Radiationresistant optical fiber amplifiers for satellite communications,” Proc. SPIE 10096, 100960H (2017).

[4] E. Kehayas, J. Edmunds, C. Palmer, C. Coopman, E. Kehayas, J. Edmunds, C. Palmer, C. Coopman, R. Webb, M. Tuci, K. Simpson, M. Welch, and L. Stampoulidis, "Space qualification of multi-channel optical fiber amplifier for low Earth," Proc. of SPIE Vol. 10524, 105240Z (2018)

[5] P. Henderson, A. Norman, J. Macdougal, P. Naylor, E. Kehayas, L. S. How, S. Lhuillier, A. Bensossan, and M. Zahir, "Space Validation of 1550nm DFB Laser Diode Module,” International Conference on Space Optics, 2018. 